\title{
3 Research Square

\section{The Correlation of Hip Joint Passive Range of Motion with Lower Limbs Injuries in Professional Soccer Players}

Elham Karaminia ( $\sim$ meek.1363@yahoo.com )

sport medicine, Iran University of Science and Technology

Hoomam Angoorani

Iran University of Medical Sciences, Department of sport medicine hazard rasool-e akram hospital- iran football Medical Assessment and Rehabilitation Center, IFMARC

Ali Mazaherinezhad

Iran university of medical center-Department of sport medicine, Hazrat-e Rasool General Hospital

\section{Bahar Hassanmirzaei}

Iran football medical Assessment and rehabilitation Center,IFMARC - Sport medicine Research Center, Neuroscience Institute Tehran university of medical Science

Navid Moghadam

Sport Medicine Research Center, Neuroscience Institute,Tehran university of Medical sciences,

\section{Original Research Article}

Keywords: Hip Range of Motion, Lower limb Injuries, Soccer

Posted Date: March 27th, 2020

DOl: https://doi.org/10.21203/rs.3.rs-19076/v1

License: (c) (1) This work is licensed under a Creative Commons Attribution 4.0 International License.

Read Full License 


\title{
The Correlation of Hip Joint Passive Range of Motion with Lower Limbs Injuries in Professional Soccer Players
}

\author{
Short title: Hip ROM correlates with injuries in Soccer
}

\begin{abstract}
Introduction: Soccer is a popular sport with a high injury rate. Lower limb injuries are accounted for $85 \%$ of injuries in professional Soccer player. It is important to identify the predisposing factors in professional Soccer players for screening players at an increased risk of injury. It is unclear whether to assume limited hip range of motion (ROM) as one of these factors this study aimed to evaluate the association between hip passive ROM and lower limb injury in Iranian professional Soccer players.

Design: This prospective cohort study was conducted on 266 male professional Soccer players (with a mean age of $24.91 \pm 4.38$ years) playing in the Iran Soccer Premium League during the 2017-2018 and 2018-2019 season. Baseline hip joint passive ROM was evaluated in all subjects. All athletes were followed for two consecutive seasons and their lower limb injuries causing at least an absent from a competitive match was reported. The association between hip ROM and the incidence of lower limb injuries was analyzed.

Results: The most common lower limb injuries were ankle and foot injuries (12.4\%), groin pain $(11.5 \%)$ and muscle strain (10.9\%), respectively. Restricted hip internal rotation (IR) was associated with a higher incidence of groin pain, ACL injury and knee injury. A significant correlation was observed between decreased hip external rotation (ER) with muscle strain and
\end{abstract}


knee injuries. The higher knee injury was also associated with reduced hip abduction. There was no association between ankle and foot injuries and hip passive ROM.

Conclusions: This study showed that hip passive ROM could be used to identify Soccer players at a higher risk of lower limb injuries. Hip rotational ROM and abduction limitation were significantly associated with the incidence of several common Soccer injuries, including groin pain, muscle strain and ACL/knee injuries and precedes their development and could be used as a screening tool for professional male soccer players.

Author Keywords: Hip Range of Motion, Lower limb Injuries, Soccer 


\section{Introduction}

Soccer is one of the most popular sport worldwide (1). According to previous studies, Soccer has a higher injury rate compared to other competitive sports. The incidence of injury is estimated to be 15 to 35 injuries per 1000 hours of training/match $(2,3)$. Soccer is a high demand sport which puts a lot of pressure and stress on lower extremity Due to high loads of running, explosive movements, directional changes, repeated kicking, and body contact. In this regard lower limb injuries accounts for $85 \%$ of injuries in a professional Soccer player. Muscle and tendon injuries are most frequently involved with strains, sprains, and contusions being the most common types of injuries $(7,8)$.

Lower limb injuries are a major concern in professional athletes as they lead to decreased performance, financial cost, and long-term absence from match and health-related problems (9). Therefore, it is important to identify and assess precisely the risk factor associated and the predictor's lower limb injuries in professional Soccer players. Several screening tools such as muscle strength assessment and balance index score has been described with conflicting results to identify players at an increased risk for injuries to prescribe individualized prevention measures $(1$

\section{There are no sources in the current document. 0,11 )}

Some studies have suggested that lower limb injuries are associated with a restricted hip range of motion (ROM) and some have not. The results are contradictory regarding this matter and it is unclear whether to assume hip ROM restriction as a predisposing anthropomorphic factor for the lower limb injuries in professional athletes. Limited studies are describing this association and the result of those studies could not be relied upon because, first, they enrolled mainly male professional football players, second, most of them are case-control studies and 
cohort studies are limited regarding this subject. And third, the few cohort studies have a short follow-up period that may influence their results $(1,6,12-17)$. A systematic review reported that there was very limited evidence on this matter prospectively. The only significant finding concerning the hip ROM was its association with groin pain in 2 retrospective high-quality studies (18). Furthermore, most of the studies concerning this topic focused only on a small portion of lower limb injuries, small sample size and short duration of follow up. Due to these shortcomings, we conducted a 2-year prospective cohort study to evaluate the association between hip passive ROM and lower limb injury in Iranian professional Soccer players. With this study, we aimed to fully evaluate the role of hip ROM as a predisposing risk factor in male professional Soccer players and to assess its association with all types of lower limb injuries.

\section{Material and methods}

\section{Study design and subjects}

This study was a prospective cohort study, approved by the ethics committee. Informed consent was obtained from all players. Three hundred professional male Soccer players playing in the Iran Soccer Premium League during the 2017-2018 and 2018-2019 season were enrolled in this study. The exclusion criteria were history of ACL tear, history of ankle sprain within the last 1 year; history of muscle strain or groin pain in the last 6 months; leaving the Iran Soccer Premium League (to either lower domestic league or abroad); and Lack of cooperation of team physician to record injuries. Thirty four excluded from this study. Twenty seven players were excluded based on their examination at the first medical assessment before the season and 8 players were excluded in the middle of the study as they left the professional league. (16 players with a history of ankle injury, 3 players with the history of ACL tear, 2 players with the injuries of other knee ligaments, 4 players with a muscle strain, 3 players with groin pain and 8 players 
due to leaving the premium League) and, the study was carried out with 266 professional male Soccer players.

\section{Measurements}

Baseline hip joint passive ROM evaluation was performed by a sports medicine specialist Using a digital goniometer mobee med (SportMed A.G. SA, Echternach, Luxembourg) with a mean of three measurements recorded. For hip flexion evaluation, the athlete was placed in the supine position. The examiner placed the hip in maximum flexion and the angle between the centerline of the femur and the examination bed was recorded. The hip extension was evaluated with subjects in the prone position and the angle between the centerline of the femur and the examination bed was recorded after placing the hip in maximum extension. Hip adduction and abduction were checked with participants in the supine position and hip extended. The examiner, one hand on the athlete's ankle, the other on the anterior superior iliac spine (ASIS), move the tested limb toward and away from the central line to evaluate adduction and abduction, respectively. The endpoint was reached when passive motion in any of the two directions started to move the pelvis. The angle between the line perpendicular to the center of the line linking the bilateral ASIS and the centerline of the femur was reported. Internal and external rotation were evaluated with the athlete in supine position and hip in neutral rotational position and flexed. The examiner, holding the knee with one hand and the foot with the other, place the knee and hip in $90^{\circ}$ of flexion. Then, the hip was rotated internally and externally with the hand on the foot and the femur immobilized with the other hand and the rotation measures were recorded using the tibial axis. All measures were reported as normal or decreased. Based on previous reports normal hip passive ROM value were considered:

$129 \pm 9^{\circ}$ for flexion, $20 \pm 11^{\circ}$ for extension, $46 \pm 8^{\circ}$ for abduction, $24 \pm 11^{\circ}$ for adduction, 32 $\pm 8^{\circ}$ for internal rotation (IR) and $38 \pm 8^{\circ}$ for external rotation (ER) $(19,20)$. 
Athletes were followed for two consecutive playing seasons and lower limb injuries, including groin and pelvic related pain, ACL injury, knee injuries, muscle strain, and ankle and foot injuries that caused at least a absent from match following the injury for the upcoming two playing seasons were reported. During the season, lower limb injuries were recorded on a daily basis by the team physicians, according to an injury checklist published by the Federation International de Soccer Association (FIFA) and were collected from the team physicians by a weekly basis. During the next year Pre competition Medical Assessment (PCMA), the incidence of lower limb injuries for the previous season was asked of all players to check for any missing injury record and the final report was made. No treatment or specific preventive measures regarding hip joint ROM were given to any athlete enrolled in this study.

\section{Statistical analysis}

The association between hip joint ROM and the incidence of lower limb injuries was analyzed using SPSS software V20, SPSS Inc., Chicago, IL, USA. The data were analyzed by descriptive statistics (frequency, mean, percent, and standard deviation) and the Chi-Square test was used for statistical comparison. The significant threshold was considered to be less than 0.05 .

\section{Results}

Two hundred sixty six professional male Soccer players enrolled in this study with a mean age of $24.91 \pm 4.38$ years. The hip joint passive ROM is demonstrated in table 10. Reduced passive hip flexion and extension ROM was observed in 229 (86.1\%) and 245 (92.1\%) of cases in the right hip and $199(74.8 \%)$ and 255 (95.5\%) of cases in the left hip, respectively. The left hip abduction was restricted in 13 subjects (4.9\%), whereas the right hip abduction was normal in all participants. Decreased hip adduction was observed in $241(90.6 \%)$ and $168(63.2 \%)$ of 266 participants in the right and left hip evaluation, respectively. Right hip internal and external rotations were restricted in $82(30.8 \%)$ and $142(53.4 \%)$ of athletes, respectively, and reduced 
left internal and external rotation was reported in $108(40.6 \%)$ and $126(47.4 \%)$ of participants, respectively.

Ankle and foot injuries were the most frequently reported lower limb injury in our study with an incidence of $12.4 \%$ (33 cases on either side) followed by groin and pelvic related pain and Muscle strain with an incidence of $11.5 \%$ and $10.9 \%$, respectively. Right and left knee injuries were observed in 22 and 18 Soccer players enrolled in the present study with an incidence of $7.5 \%$. ACL tear was the least common lower limb with an incidence of $2.6 \%$.

The association between passive hip ROM and either injury is demonstrated in table 3-7. According to table 3, only right IR was significantly associated with groin pain in Soccer players with a p-value of 0.04 . As it's shown, groin pain was more frequent in subjects with restricted right IR (an incidence of $18.3 \%$ compared to 9.8\%). Reduced right ER and normal left adduction were significantly associated with the occurrence of muscle strain with a p-value of 0.05 and 0.02 , respectively (an incidence of $16.9 \%$ and $13.3 \%$ compared to $9.7 \%$ and $5.4 \%$, respectively) (Table 4). ACL injury was observed in $15.4 \%$ of subjects with reduced left hip abduction, whereas its incidence was $2 \%$ in athletes with normal left abduction ROM and this difference was significant with a p-value of 0.04 (Table 5). We observed a significant correlation between the occurrence of knee injury with restricted left abduction, IR and ER, all with p-value $<0.05(23.1 \%, 12 \%$, and $11.1 \%$ compared to $5.9 \%, 3.2 \%$, and $2.9 \%$, respectively) (Table 6). According to table 7, there was no significant association between hip passive ROM and ankle and foot injuries in this study.

\section{Discussion}

Although hip ROM evaluation is a vital part of clinical assessment to determine treatment and returning to play in professional Soccer players, there is conflicting evidence of restrained ROM being a predisposing risk factor for lower limb injuries in this population (21). Evaluation 
the risk factors in hip and pelvic area for lower extremity injuries in sport, the iliopsoas muscle tightness and a decreased hip rotation were suggested as possible risk factors (Krivickas, Ibrahim, Gomes, Bradley, Shah) This study aimed to evaluate hip passive ROM in professional male soccer players and to check whether its restriction could be associated with a higher incidence of lower limb injuries or not.

According to the findings of the present study, except for ankle and foot injuries, a higher incidence of common lower limb injuries, i.e. groin and pelvic related pain, muscle strain, ACL tear, and knee injuries, was associated with reduced hip passive ROM. As demonstrated, reduced passive IR was associated with a higher incidence of groin pain, ACL injury and knee injuries. A significant correlation was observed between decreased hip ER with muscle strain and knee injuries. The higher knee injury rate was also associated with reduced hip abduction. Of note, the most common lower limb injuries in the present study were ankle and foot injuries (12.4\%), groin pain (11.5\%) and muscle strain (10.9\%), respectively. Several studies have been conducted to investigate the effect of hip ROM on the occurrence of different lower limb injuries $(6,12)$.

Krivickas and Feinberg showed that there is a relationship between iliopsoas muscle tightness and overuse injuries in the knee (22).

In the present study, the groin and pelvic related pain was associated with right hip IR limitation. The association between groin pain and hip ROM has been studied by different authors. In a systematic review of 11 studies by Tak et al. in 2017 (6), the association between hip ROM and groin and pelvic related pain was evaluated. It was reported that a pre-season total hip rotation $\left(\mathrm{ER}+\mathrm{IR}\right.$ with hip and knee flexed to $\left.90^{\circ}\right)$ of $<85^{\circ}$ has a strong association with the incidence of groin and pelvic related pain in professional athletes. On the other hand, strong evidence suggested that reduced IR, abduction and extension were not associated with the incidence of groin pain. Kloskowsk et al. in their meta-analysis (18), reported that among 
ROM, strong evidence was observed between total hip ER (sum of both hip ER) with sportrelated groin pain (SRGP). This associated was observed evaluating retrospectively They suggested that increasing hip IR and ER should be considered during the rehabilitation of athletes with SRGP and hip rotation ROM is not suitable for screening and identifying players at risk. According to Mosler et al. (23), hip ER could be used to differentiate players with and without SRGP (strong evidence when measuring with hip in the neutral position and moderate evidence when evaluating with hip and knee at $90^{\circ}$ of flexion). They Also demonstrated that athletes with SRGP had a lower hip ROM by $3.6 \mathrm{~cm}(1.3-5.8 \mathrm{~cm})$ evaluated by the bent knee fall out (BKFO), combined evaluation of hip flexion, abduction and external rotation. Verrall et al. (14) followed 29 elite Australian Soccer players for 2 consecutive seasons for the occurrence of chronic groin injury (CGI) and reported that total hip ROM (sum of total hip ER and IR) was associated with groin injury and its restriction precede groin injury development $\left(83.13^{\circ}\right.$ compared to $103.18^{\circ}$ with a rate ratio (IR) of 0.90 and a p-value of 0.03$)$. In Nevin et al. study of 36 Gaelic Soccer players (24), those players with longstanding groin injury had a significantly lower BKFO, hip IR, and ER, bilaterally (all with p-value $<0.05$ ). On the contrary, Mosler et al. (25) reported that among male professional soccer players, aged $18 \geq$ years in the Qatar Stars League, none of the hip ROMs were significantly associated with the risk of SRGP and suggested that there is a little indication to include ROM measures in preseason screening. Cheng et al. (26) conducted a 5-year cohort study on 94 female Soccer players and reported that baseline hip ROMs values were not predictive for lower limb injury occurrence. This difference with the present study could be interpreted based on their different study populations. Although both studies were conducted among professional Soccer players, Cheng et al. enrolled adolescent female athletes with a mean age of $14.6 \pm 1.8$ years compared to the present study subjects which were male players with a mean age of $24.91 \pm 4.38$ years. 
The ration for the association of hip joint restriction and CGI and groin pain is described in several studies. It is hypothesized that in athletes with a restricted hip joint ROM, greater stress is implied across the superior pubic ramus, pubic symphysis and surrounding soft tissues leading to increased forces in this vulnerable area, therefore, the increased likelihood of development of CGI (27-29). Furthermore, for adequate load absorption during activities requiring change of directions, sufficient hip ROM is vital, therefore, reduced hip ROM could be a critical factor for SRGP (30). Additionally, another suggested mechanism for SRGP is Femoroacetabular Impingement (FAI) which could be manifested through reduced hip IR (31). Kapron et al. (32) suggested that Soccer players with reduced hip IR and groin pain should be evaluated for underlying Cam FAI abnormalities.

Other predisposing factors have been also described in the literature for SRGP. According to Kloskowsk et al. (18) and Mosler et al. (23) meta-analyses, the strongest predisposing risk factor for the incidence of SRGP is reduced hip adductor strength evaluated by squeeze and unilateral resisted adduction tests, which should be considered as a screening method in preseason assessments. Mosler at al. (25) in their 2-year cohort study reported that eccentric hip adduction strength was associated with hip/groin injuries. According to Ryan et al. Systematic review (33), previous groin/hip injury is the most predominant risk factor for groin injuries in field-based sports followed by older age and adductor weakness. In Larson et al. study of National Soccer League (NFL) players (34), alpha angle $>55^{\circ}$ (larger cam deformities) was the only independent predictor of SRGP with $\mathrm{p}=0.01$ (with hip symptom incidence of $37.2 \%$ compared to $20.5 \%$ ).

Several studies have been carried out to evaluate the association between ACL injuries and hip ROM. In the present study, decreased left hip passive IR and abduction was associated with ACL injuries among Soccer player. In Tainaka et al. study (15), the association between hip active ROM limitation and non-contact ACL injuries was evaluated. According to their 
findings, only limited hip IR and ER were significantly related to the development of ACL injury with an odds ratio $(\mathrm{OR})$ for a $10^{\circ}$ increase $=0.18$ and 0.23 , for hip IR and ER, respectively, both with a p-value of $<0.01$. IR and ER were $35.0 \pm 9.1^{\circ}$ and $45.7 \pm 6.1^{\circ}$ in the participants with non-contact ACL injury compared to $50.2 \pm 7.2^{\circ}$ and $56.3 \pm 6.8^{\circ}$ in the control group, respectively. In VandenBerg study of 50 athletes (13), patients with an injured ACL has a lower IR compared to control group $\left(23.4 \pm 7.6^{\circ}\right.$ compared to $30.4 \pm 10.4^{\circ}$ with $p$-value $<$ 0.01). They suggested that a $10^{\circ}$ increase of hip IR could lead to a decreased risk of ACL injury with an OR of 0.419. In Gomes et al. study (12), strong relation was observed between the incidence of non-contact ACL injury with restricted hip rotational ROM (either IR, ER or sum of ER + IR). Furthermore, according to a systematic review carried by Boutris et al. (35), a significant association was observed between ACL injuries and hip rotational restriction $\left(>10^{\circ}\right.$ of IR loss or $>20^{\circ}$ of ER or IR+ER loss). Additionally, Bedi et al. (36) reported that in 224 NFL players, the hip IR reduction was correlated with a statistically significant increased risk of ACL injury in the ipsilateral or contralateral knee $(\mathrm{OR}=0.95, \mathrm{p}<0.01)$.

It has been demonstrated that non-contact ACL injuries are associated with the valgus positioning of the knee joint. The hip joint rotational ROM is regarded as a buffer to reduce and divert the loading stress on the knee joint in this position. The restriction of hip ROM will impose strong rotational force on the knee joint, leading to the increased strain of the ACL accompanying the risk of ACL fatigue failure and therefore, a higher rate of ACL tear $(13,15$, $35,37)$. In line with this potential mechanism of ACL injury, several cadaveric studies have demonstrated that peak ACL strain was inversely related to the range of internal rotation of the femur (38).

Previous studies have reported several structural risk factors preceding ACL injury, including Q-angle, genu recurvatum, and navicular drop. Contrary to these factors which are hard to improve due to their nature, hip ROM restriction could be easily addressed with stretching 
technique, therefore adding this training is a good, feasible strategy to avoid ACL injury in professional athletes $(15,39)$.

Our result showed a significant relationship between limited right hip ER with muscle injuries. Other authors reported similar results. Ibrahim et al. (1) conducted a study on 120 professional Soccer players playing in Australia. They reported that the pre-season hip total rotational ROM $(\mathrm{IR}+\mathrm{ER})$ in players with an adductor strain during play season was lower compared to normal participants $\left(44.7^{\circ}\right.$ compared to $53.7^{\circ}$ with p-value $\left.=0.03\right)$. According to Bradley et al. study (5), Soccer players with lower pre-season ROM in the hip flexors and knee flexors had a statistically higher risk of muscle strain injuries to these muscle groups during a competitive season.

In a recent study Shah et al suggest that muscle tightness in the transverse plane about the vertical axis may be more important for predicting lower extremity injuries and decrease hip internal rotation is associated with in season lower extremity injuries in soccer players (40)

It should be noted that according to table 1, most participants had reduced hip joint extension, flexion, adduction, and ER. Previous studies have reported that professional sport training could lead to a capsular response responsible for restriction of hip joint ROM in a large portion of professional athletes, but further research is required for identifying why some athletes are affected whereas others are not $(14,27)$.

Of note, we observed differences evaluating either right or left hip ROM association with injuries as groin pain and muscle strain were associated with right hip ROM restriction, whereas left hip ROM shortcoming was significantly related to ACL and knee injuries. According to the literature, the imbalance in the strength of the two lower extremities leg dominance or leg dominancy places additional stress on the weaker leg and is a vital predisposing factor during cutting/ landing activities leading to a higher rate of knee/ACL (41). Although the leg dominancy and the rate of injury compared to this variable were not 
mentioned in our study, the ACL/knee injury was significant on the left side, the assumed dominant leg in the majority of the general population in line with the above-mentioned finding.

\section{Strengths and limitation}

The current study was a 2-year cohort evaluation compared to other similar studies which had a shorter durations, mainly 1 year and less. More over this study included a higher population of professional athletes compared to other studies. Furthermore, all hip ROMs were assessed in the present. All the ROMs were recorded using a digital goniometer. This method will reduce the human based errors using the manual goniometer in this regard. Not only focusing on one injury, this study was able to evaluate the hip ROM with all lower extremity injuries in professional Soccer players.

One limitation of this study is that we relied on information to record further damage, which was available to us through medical staff and was not possible to examine all of the injured players during the season. Another limitations of this study was not to include female soccer players. Femoroacetabular impingement (FAI) could be a reason for reduced internal rotation of the hip. Considering the high prevalence of FAI in soccer, but none of the players reported any precise diagnosis of such situation. There was no radiological evaluation done on the players to exclude those with FAI in this study because of ethical issues of unnecessary x-ray predisposition.

\section{Conclusions}

This study showed that hip passive ROM could be used to identify those Soccer players at a higher risk of lower limb injuries. Hip rotational ROM and abduction stiffness were significantly associated with the incidence of several common Soccer injuries, including groin and pelvic related pain, muscle strain and ACL/knee injuries. Based on a direct impact of 
injuries on professional career of a soccer player and a club performance, Hip joint ROM could be used as a screening tool for professional male soccer players.
Abbreviations
IR: Internal rotation
ER: External rotation
Flex: Flexion
Ext: Extension
ROM: Range of motion

\section{Ethical Approval and Consent to participate}

All research participants have signed and approved the participation consent form.

\section{Consent for publication}

The Author transfers to Springer (respective to owner if other than Springer and for U.S.

government employees: to the extent transferable) the non-exclusive publication rights and he warrants that his/her contribution is original and that he/she has full power to make

\section{Availability of data and materials}

All article reviews and data are available.

\section{Funding}

No funding has been received. 


\section{Authors' contributions}

Corresponding Author: Dr. Elham Karaminia

Other Authors contributed equally

\section{Conflict of Interest}

No conflicts.

\section{Acknowledgments}

Authorship Conformation Form Acknowledgments We thank all physicians in the Soccer Premium League and the IFMARK complex for their cooperation.

\section{Author's name}

Elham Karaminia, Department of Sport Medicine, Hazrat Rasool-e Akram Hospital- Iran University of medical sciences, Tehran, Iran.

Hooman Angoorani, Department of sport medicine, Hazrat rasool-e akram hospital, Tehran, Iran

Iran university of medical sciences, Tehran, Iran- Iran Football Medical Assessment and Rehabilitation Center, IFMARC, Tehran, Iran

Ali Mazaherinezhad, Department of sport medicine, Hazrat rasool-e akram hospital- Iran University of Medical Sciences, Tehran, Iran

Bahar Hassanmirzaei, Iran Football Medical Assessment and Rehabilitation Center, IFMARC, Tehran, Iran Sports Medicine Research Center, Neuroscience Institute, Tehran University of Medical Sciences, Tehran, Iran

Navid Moghadam, Sports Medicine Research Center, Neuroscience Institute, Tehran University of Medical Sciences, Tehran, Iran 


\section{References}

1. Ibrahim A, Murrell GA, Knapman P. Adductor strain and hip range of movement in male professional soccer players. Journal of Orthopaedic Surgery. 2007 Apr; 15(1):46-9.

2. Wong P, Hong Y. Soccer injury in the lower extremities. British journal of sports medicine. 2005 Aug 1; 39(8):473-82.

3. Herrero H, Salinero JJ, Del Coso J. Injuries among Spanish male amateur soccer players: a retrospective population study. The American journal of sports medicine. 2014 Jan; 42(1):78-85.

4. Arnason A, Sigurdsson SB, Gudmundsson A, Holme I, Engebretsen L, Bahr R. Risk factors for injuries in football. The American journal of sports medicine. 2004 Mar; 32(1_suppl):516.

5. Bradley PS, Portas MD. The relationship between preseason range of motion and muscle strain injury in elite soccer players. Journal of Strength and Conditioning Research. 2007 Nov 1; 21(4):1155.

6. Tak I, Engelaar L, Gouttebarge V, Barendrecht M, Van den Heuvel S, Kerkhoffs G, Langhout R, Stubbe J, Weir A. Is lower hip range of motion a risk factor for groin pain in athletes? A systematic review with clinical applications. British journal of sports medicine. 2017 Nov 1; 51(22):1611-21.

7. Pangrazio O, Forriol F. Epidemiology of soccer players' traumatic injuries during the 2015 America Cup. Muscles, ligaments and tendons journal. 2016 Jan; 6(1):124.

8. Carling C, Orhant E, LeGall F. Match injuries in professional soccer: inter-seasonal variation and effects of competition type, match congestion and positional role. International journal of sports medicine. 2010 Apr; 31(04):271-6.

9. Bakken A, Targett S, Bere T, Eirale C, Farooq A, Mosler AB, Tol JL, Whiteley R, Khan $\mathrm{KM}$, Bahr R. Muscle strength is a poor screening test for predicting lower extremity injuries 
in professional male soccer players: A 2-year prospective cohort study. The American journal of sports medicine. 2018 May; 46(6):1481-91.

10. Bahr R. Why screening tests to predict injury do not work — and probably never will...: a critical review. Br J Sports Med. 2016 Jul 1; 50(13):776-80.

11. Schnurrer-Luke Vrbanić T, Ravlić-Gulan J, Gulan G, Matovinović D. Balance index score as a predictive factor for lower sports results or anterior cruciate ligament knee injuries in Croatian female athletes-preliminary study. Collegium antropologicum. 2007 Jan 4; 31(1):253-8.

12. Gomes JL, de Castro JV, Becker R. Decreased hip range of motion and noncontact injuries of the anterior cruciate ligament. Arthroscopy: The Journal of Arthroscopic \& Related Surgery. 2008 Sep 1; 24(9):1034-7.

13. VandenBerg C, Crawford EA, Enselman ES, Robbins CB, Wojtys EM, Bedi A. Restricted hip rotation is correlated with an increased risk for anterior cruciate ligament injury. Arthroscopy: The Journal of Arthroscopic \& Related Surgery. 2017 Feb 1; 33(2):317-25.

14. Verrall GM, Slavotinek JP, Barnes PG, Esterman A, Oakeshott RD, Spriggins AJ. Hip joint range of motion restriction precedes athletic chronic groin injury. Journal of Science and Medicine in Sport. 2007 Dec 1; 10(6):463-6.

15. Tainaka K, Takizawa T, Kobayashi H, Umimura M. Limited hip rotation and non-contact anterior cruciate ligament injury: A case-control study. The Knee. 2014 Jan 1; 21(1):8690.

16. Weir A, Brukner P, Delahunt E, Ekstrand J, Griffin D, Khan KM, Lovell G, Meyers WC, Muschaweck U, Orchard J, Paajanen H. Doha agreement meeting on terminology and definitions in groin pain in athletes. Br J Sports Med. 2015 Jun 1; 49(12):768-74.

17. Whittaker JL, Small C, Maffey L, Emery CA. Risk factors for groin injury in sport: an updated systematic review. Br J Sports Med. 2015 Jun 1; 49(12):803-9. 
18. Kloskowska P, Morrissey D, Small C, Malliaras P, Barton C. Movement patterns and muscular function before and after onset of sports-related groin pain: a systematic review with meta-analysis. Sports Medicine. 2016 Dec 1; 46(12):1847-67.

19. Mosler AB, Crossley KM, Thorborg K, Whiteley RJ, Weir A, Serner A, Hölmich P. Hip strength and range of motion: normal values from a professional football league. Journal of science and medicine in sport. 2017 Apr 1;20(4):339-43.

20. HALLAİELİ H, URUI V, Uysal HH, ÖZDEN R, HALLAİELİ İ, Soyuer F, Parpucu TI, Yengil E, CAVLAK U. Normal hip, knee and ankle range of motion in the Turkish population. Acta Orthop Traumatol Turc. 2014; 48(1):37-42.

21. Whittaker JL, Small C, Maffey L, Emery CA. Risk factors for groin injury in sport: an updated systematic review. Br J Sports Med. 2015 Jun 1; 49(12):803-9.

22. Krivickas LS, Feinberg JH. Lower extremity injuries in college athletes: relation between ligamentous laxity and lower extremity muscle tightness. Archives of physical medicine and rehabilitation. 1996 Nov 1; 77(11):1139-43.

23. Mosler AB, Weir A, Hölmich P, Crossley KM. Which factors differentiate athletes with hip/groin pain from those without? A systematic review with meta-analysis. Br J Sports Med. 2015 Jun 1; 49(12):810-.

24. Nevin F, Delahunt E. Adductor squeeze test values and hip joint range of motion in Gaelic football athletes with longstanding groin pain. Journal of science and medicine in sport. 2014 Mar 1; 17(2):155-9.

25. Mosler AB, Weir A, Serner A, Agricola R, Eirale C, Farooq A, Bakken A, Thorborg K, Whiteley RJ, Hölmich P, Bahr R. Musculoskeletal Screening Tests and Bony Hip Morphology Cannot Identify Male Professional Soccer Players at Risk of Groin Injuries: A 2-Year Prospective Cohort Study. The American journal of sports medicine. 2018 May; 46(6):1294-305. 
26. Cheng AL, Merlo JA, Hunt D, Yemm T, Brophy RH, Prather H. Are Hip Physical Examination Findings Predictive of Future Lower-Body Injury Rates in Elite Adolescent Female Soccer Athletes at Minimum 5-Year Follow-Up. Journal of sport rehabilitation. 2019 Apr 29; 1(aop):1-7.

27. Verrall GM, Hamilton IA, Slavotinek JP, Oakeshott RD, Spriggins AJ, Barnes PG, Fon GT. Hip joint range of motion reduction in sports-related chronic groin injury diagnosed as pubic bone stress injury. Journal of Science and Medicine in Sport. 2005 Mar 1; 8(1):7784.

28. Fricker PA. Management of groin pain in athletes. British journal of sports medicine. 1997 Jun $1 ; 31(2): 97-101$.

29. Williams JG. Limitation of hip joint movement as a factor in traumatic osteitis pubis. British journal of sports medicine. 1978 Sep; 12(3):129.

30. L'hermette M, Polle G, Tourny-Chollet C, Dujardin F. Hip passive range of motion and frequency of radiographic hip osteoarthritis in former elite handball players. British journal of sports medicine. 2006 Jan 1; 40(1):45-9.

31. Siebenrock KA, Kaschka I, Frauchiger L, Werlen S, Schwab JM. Prevalence of cam-type deformity and hip pain in elite ice hockey players before and after the end of growth. The American journal of sports medicine. 2013 Oct; 41(10):2308-13.

32. Kapron AL, Anderson AE, Peters CL, Phillips LG, Stoddard GJ, Petron DJ, Toth R, Aoki SK. Hip internal rotation is correlated to radiographic findings of cam femoroacetabular impingement in collegiate football players. Arthroscopy: The Journal of Arthroscopic \& Related Surgery. 2012 Nov 1; 28(11):1661-70.

33. Ryan J, DeBurca N, Mc Creesh K. Risk factors for groin/hip injuries in field-based sports: a systematic review. Br J Sports Med. 2014 Jul 1; 48(14):1089-96. 
34. Larson CM, Sikka RS, Sardelli MC, Byrd JT, Kelly BT, Jain RK, Giveans MR. Increasing alpha angle is predictive of athletic-related "hip" and "groin" pain in collegiate National football League prospects. Arthroscopy: The Journal of Arthroscopic \& Related Surgery. 2013 Mar 1; 29(3):405-10.

35. Boutris N, Byrne RA, Delgado DA, Hewett TE, McCulloch PC, Lintner DM, Harris JD. Is there an association between noncontact anterior cruciate ligament injuries and decreased hip internal rotation or radiographic femoroacetabular impingement? A systematic review. Arthroscopy: The Journal of Arthroscopic \& Related Surgery. 2018 Mar 1; 34(3):943-50.

36. Bedi A, Warren RF, Wojtys EM, Oh YK, Ashton-Miller JA, Oltean H, Kelly BT. Restriction in hip internal rotation is associated with an increased risk of ACL injury. Knee Surgery, Sports Traumatology, Arthroscopy. 2016 Jun 1; 24(6):2024-31.

37. Renstrom P, Ljungqvist A, Arendt E, Beynnon B, Fukubayashi T, Garrett W, Georgoulis T, Hewett TE, Johnson R, Krosshaug T, Mandelbaum B. Non-contact ACL injuries in female athletes: an International Olympic Committee current concepts statement. British journal of sports medicine. 2008 Jun 1; 42(6):394-412.

38. Nakano N, Bartlett J, Khanduja V. Is restricted hip movement a risk factor for anterior cruciate ligament injury? Journal of Orthopaedic Surgery. 2018 Sep 24; 26(3):2309499018799520.

39. Kibler WB, Chandler TJ. Range of motion in junior tennis players participating in an injury risk modification program. Journal of science and medicine in sport. 2003 Mar 1; 6(1):5162.

40. Shah SS, Testa EJ, Gammal I, Sullivan J, Gerland RW, Goldstein J, Sheridan B, Mashura M, Shah AS, Goodwillie A, Cohn RM. Hip Range of Motion: Which Plane of Motion Is More Predictive of Lower Extremity Injury in Elite Soccer Players? A Prospective Study. Journal of surgical orthopaedic advances. 2019; 28(3):201-8. 
41. Read PJ, Oliver JL, Croix MB, Myer GD, Lloyd RS. Neuromuscular risk factors for knee and ankle ligament injuries in male youth soccer players. Sports Medicine. 2016 Aug 1; 46(8):1059-66. 


\section{Supplementary Files}

This is a list of supplementary files associated with this preprint. Click to download.

- IFMARCPCMA.pdf 